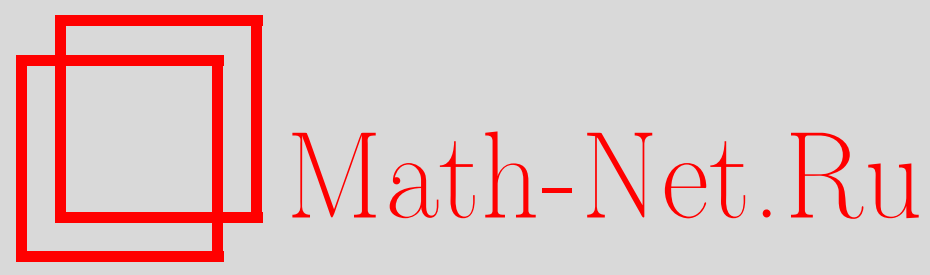

В. В. Дмитриева, Точечно-инвариантные классы обыкновенных дифференциальных уравнений третьго порядка, Матем. заметки, 2001, том 70, выпуск 2, 195-200

DOI: https://doi.org/10.4213/mzm733

Использование Общероссийского математического портала Math-Net.Ru подразумевает, что вы прочитали и согласны с пользовательским соглашением http://www.mathnet.ru/rus/agreement

Параметры загрузки:

IP : 3.93 .64 .190

26 апреля 2023 г., $14: 21: 42$

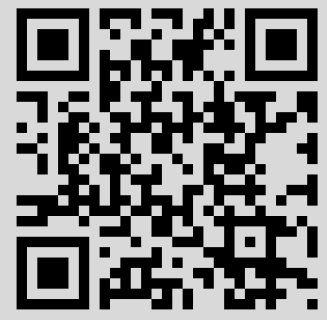




\section{ТОЧЕЧНО-ИНВАРИАНТНЫЕ КЛАССЫ ОБЫКНОВЕННЫХ ДИФФЕРЕНЦИАЛЬНЫХ УРАВНЕНИЙ ТРЕТЬГО ПОРЯДКА}

\section{В. В. Дмитриева}

Рассмотрено действие точечных преобразований общего вида на обыкновенные диффференциальные уравнения третьего порядка, разрешенные относительно старшей производной. Выделены инвариантные классы уравнений относительно данных преобразований.

Библиография: 25 названий.

1. Введение. Рассмотрим класс обыкновенных дифференциальных уравнений третьего порядка, разрешенных относительно старшей производной

$$
y^{\prime \prime \prime}=f\left(x, y, y^{\prime}, y^{\prime \prime}\right)
$$

Точечные преобразования общего вида

$$
\left\{\begin{array}{l}
\tilde{x}=\tilde{x}(x, y), \\
\tilde{y}=\tilde{y}(x, y)
\end{array}\right.
$$

переводят уравнение (1.1) в новое уравнение

$$
\tilde{y}^{\prime \prime \prime}=g\left(\tilde{x}, \tilde{y}, \tilde{y}^{\prime}, \tilde{y}^{\prime \prime}\right)
$$

Выберем специальньй класс уравнений (1.1), у которых правая часть $f$ принадлежит некоторому заданному семейству функций $\mathscr{F}($ т.е. $f \in \mathscr{F})$.

ОПРЕДЕЛЕниЕ 1. Если для любой фунцкции $f \in \mathscr{F}$ правая часть преобразованного уравнения (1.3) также принадлежит $\mathscr{F}$, т.е. $g \in \mathscr{F}$, то данный класс уравнений называется инвариантным или замкнуты.м классом относительно преобразований (1.2).

Как правило, исследуюся специальные классы уравнений (1.1) с полиномиальной или рациональной по производньм $y^{\prime}$ и $y^{\prime \prime}$ правьми частями.

В конце XIX века Лиувилль [1], Ли [2], [3] и Трессе [4], [5] исследовали класс уравнений с полиномиальной по $y^{\prime}$ правой частью

$$
y^{\prime \prime}=P(x, y)+3 Q(x, y) y^{\prime}+3 R(x, y){y^{\prime}}^{2}+S(x, y) y^{\prime 3}
$$

Работа выполнена при финансовой поддержке Российского фонда фундаментальных исследований, грант № 00-01-00068. 
Этот класс уравнений замкнут относительно точечных преобразований общего вида (1.2). Картан ассоциировал уравнения данного класса с уравнениями геодезических в пространствах проективной связности [6]-[9].

Замкнутость класса уравнений (1.4) отмечалась также в работах Томсена [10], Гриссома и др. [11], Ибрагимова [12], Дрюма [13]-[15], Бордаг и Дрюма [16], Бордаг и Бабича [17], [18], Романовского [19], Баско и Матсумото [20], Гусятниковой и Юмагужина [21], Шарипова и Дмитриевой [22]-[24].

В [25] Шарипов и Михайлов рассмотрели другой замкнутьй класс уравнений второго порядка:

$$
y^{\prime \prime}=\frac{P(x, y)+4 Q(x, y) y^{\prime}+6 R(x, y) y^{\prime 2}+4 S(x, y) y^{\prime 3}+L(x, y) y^{4}}{Y(x, y)-X(x, y) y^{\prime}} .
$$

Он получил название точечного расширения класса (1.4).

Более сложньй замкнутый класс обыкновенных дифференциальных уравнений второго порядка упомянут Бордаг в [17]:

$$
y^{\prime 2}=P_{5}\left(y^{\prime} ; x, y\right)
$$

где функция $P_{5}$ есть полином пятого порядка по производной $y^{\prime}$.

2. Точечные преобразования производных. Обозначим

$$
S=\left\|\begin{array}{ll}
x_{1.0} & x_{0.1} \\
y_{1.0} & y_{0.1}
\end{array}\right\|, \quad T=\left\|\begin{array}{cc}
\tilde{x}_{1.0} & \tilde{x}_{0.1} \\
\tilde{y}_{1.0} & \tilde{y}_{0.1}
\end{array}\right\| .
$$

В формулах (2.1) $S$ - матрица перехода от координат $(x, y)$ к координатам $(\tilde{x}, \tilde{y})$, а $T$ - матрища обратного перехода от координат $(\tilde{x}, \tilde{y})$ к координатам $(x, y)$. Точечное преобразование (1.2) считаем невырожденньп, поэтому $\operatorname{det} S \neq 0$. Здесь и далее под обозначением $\Phi_{i . j}$ понимаем частную производную $\partial^{i+j} \Phi / \partial x^{i} \partial y^{j}$. Так

$$
x_{1.0}=\frac{\partial x(\tilde{x}, \tilde{y})}{\partial \tilde{x}} .
$$

При замене координат производная $y^{\prime}$ преобразуется по правилу

$$
y^{\prime}=\frac{y_{1.0}+y_{0.1} \tilde{y}^{\prime}}{x_{1.0}+x_{0.1} \tilde{y}^{\prime}} .
$$

Для второй производной $y^{\prime \prime}$ аналогичное преобразование имеет более сложньй вид:

$$
\begin{aligned}
y^{\prime \prime}= & \frac{y_{2.0}+2 y_{1.1} \tilde{y}^{\prime}+y_{0.2} \tilde{y}^{\prime 2}+y_{0.1} \tilde{y}^{\prime \prime}}{\left(x_{1.0}+x_{0.1} \tilde{y}^{\prime}\right)^{2}} \\
& -\frac{\left(y_{1.0}+y_{0.1} \tilde{y}^{\prime}\right)\left(x_{2.0}+2 x_{1.1} \tilde{y}^{\prime}+x_{0.2} \tilde{y}^{2}+x_{0.1} \tilde{y}^{\prime \prime}\right)}{\left(x_{1.0}+x_{0.1} \tilde{y}^{\prime}\right)^{3}} .
\end{aligned}
$$

Запишем закон преобразования третьей производной в виде

$$
\begin{aligned}
y^{\prime \prime \prime}= & \frac{a_{1} \tilde{y}^{\prime \prime \prime}+a_{2} \tilde{y}^{\prime \prime 2}+a_{3} \tilde{y}^{\prime \prime} \tilde{y}^{2}+a_{4} \tilde{y}^{\prime \prime} \tilde{y}^{\prime}+a_{5} \tilde{y}^{\prime \prime}}{\left(x_{1.0}+x_{0.1} \tilde{y}^{\prime}\right)^{5}} \\
& +\frac{a_{6} \tilde{y}^{\prime 5}+a_{7} \tilde{y}^{\prime 4}+a_{8} \tilde{y}^{\prime 3}+a_{9} \tilde{y}^{\prime 2}+a_{10} \tilde{y}^{\prime}+a_{11}}{\left(x_{1.0}+x_{0.1} \tilde{y}^{\prime}\right)^{5}} .
\end{aligned}
$$


За исключением коэффициента $a_{1}$, все коэффициенты $a_{2}, \ldots, a_{11}$ в $(2.4)$ суть функции от $x, y$ и их производных по $\tilde{x}, \tilde{y}$. Выпишем для них явные формулы.

Коэффициент при $\tilde{y}^{\prime \prime \prime}$ :

$$
a_{1}=-\left(x_{1.0}+x_{0.1} \tilde{y}^{\prime}\right)\left(y_{1.0} x_{0.1}-x_{1.0} y_{0.1}\right) .
$$

Коэффициент при $\tilde{y}^{\prime \prime 2}$ :

$$
a_{2}=3 x_{0.1}\left(y_{1.0} x_{0.1}-x_{1.0} y_{0.1}\right) .
$$

Коэффициент при $\tilde{y}^{\prime \prime} \tilde{y}^{\prime 2}$ :

$a_{3}=-6 y_{0.1} x_{1.0} x_{0.2}-3 y_{1.1} x_{0.1}^{2}+3 y_{1.0} x_{0.2} x_{0.1}+3 y_{0.2} x_{1.0} x_{0.1}+3 y_{0.1} x_{1.1} x_{0.1}$.

Коэффициент при $\tilde{y}^{\prime \prime} \tilde{y}^{\prime}$ :

$a_{4}=9 y_{1.0} x_{0.1} x_{1.1}-3 y_{2.0} x_{0.1}^{2}+3 y_{0.1} x_{2.0} x_{0.1}+3 y_{0.2} x_{1.0}{ }^{2}-3 y_{1.0} x_{0.2} x_{1.0}-9 y_{0.1} x_{1.1} x_{1.0}$.

Коэффициент при $\tilde{y}^{\prime \prime}$ :

$a_{5}=-3 y_{1.0} x_{1.1} x_{1.0}+6 y_{1,0} x_{2.0} x_{0.1}-3 y_{0.1} x_{1.0} x_{2.0}+3 y_{1.1} x_{1.0}{ }^{2}-3 y_{2.0} x_{1.0} x_{0.1}$.

Коэффициент при $\tilde{y}^{\prime 5}$ :

$$
a_{6}=3 y_{0.1} x_{0.2}{ }^{2}-3 y_{0.2} x_{0.1} x_{0.2}+y_{0.3} x_{0.1}{ }^{2}-y_{0.1} x_{0.3} x_{0.1} .
$$

Коэффициент при $\tilde{y}^{\prime 4}$ :

$$
\begin{aligned}
a_{7}= & -3 y_{0.1} x_{1.2} x_{0.1}-3 y_{0.2} x_{1.0} x_{0.2}-y_{0.1} x_{0.3} x_{1.0} \\
& -6 y_{1.1} x_{0.2} x_{0.1}+2 y_{0.3} x_{1.0} x_{0.1}+3 y_{1.0} x_{0.2}{ }^{2} \\
& -y_{1.0} x_{0.3} x_{0.1}-6 y_{0.2} x_{1.1} x_{0.1}+12 y_{0.1} x_{1.1} x_{0.2}+3 y_{1.2} x_{0.1}{ }^{2} .
\end{aligned}
$$

Коэффициент при $\tilde{y}^{\prime 3}$ :

$$
\begin{aligned}
a_{8}= & -3 y_{0.1} x_{2.1} x_{0.1}-6 y_{0.2} x_{1.1} x_{1.0}-y_{1.0} x_{0.3} x_{1.0} \\
& -3 y_{0.1} x_{1.2} x_{1.0}+12 y_{0.1} x_{1.1}{ }^{2}-3 y_{0.2} x_{2.0} x_{0.1} \\
& -3 y_{2.0} x_{0.2} x_{0.1}-6 y_{1.1} x_{0.2} x_{1.0}+6 y_{0.1} x_{2.0} x_{0.2} \\
& -12 y_{1.1} x_{0.1} x_{1.1}+6 y_{1.2} x_{1.0} x_{0.1}+y_{0.3} x_{1.0}{ }^{2} \\
& +3 y_{2.1} x_{0.1}{ }^{2}-3 y_{1.0} x_{1.2} x_{0.1}+12 y_{1.0} x_{1.1} x_{0.2} .
\end{aligned}
$$

Коэффициент при $\tilde{y}^{2}$ :

$$
\begin{aligned}
a_{9}= & -3 y_{1.0} x_{1.2} x_{1.0}+12 y_{0.1} x_{2.0} x_{1.1}+3 y_{1.2} x_{1.0}{ }^{2} \\
& +y_{3.0} x_{0.1}{ }^{2}-6 y_{2.0} x_{1.1} x_{0.1}-3 y_{0.1} x_{2.1} x_{1.0} \\
& -6 y_{1.1} x_{2.0} x_{0.1}-3 y_{1.0} x_{2.1} x_{0.1}-y_{0.1} x_{3.0} x_{0.1} \\
& -3 y_{0.2} x_{2.0} x_{1.0}+6 y_{1.0} x_{2.0} x_{0.2}+6 y_{2.1} x_{1.0} x_{0.1} \\
& -12 y_{1.1} x_{1.0} x_{1.1}+12 y_{1.0} x_{1.1}^{2}-3 y_{2.0} x_{0.2} x_{1.0} .
\end{aligned}
$$

Коэффициент при $\tilde{y}^{\prime}$ :

$$
\begin{aligned}
a_{10}= & -3 y_{2.0} x_{0.1} x_{2.0}+12 y_{1.0} x_{2.0} x_{1.1}-6 y_{2.0} x_{1.1} x_{1.0} \\
& -6 y_{1.1} x_{2.0} x_{1.0}-3 y_{1.0} x_{2.1} x_{1.0}-y_{0.1} x_{3.0} x_{1.0} \\
& +2 y_{3.0} x_{1.0} x_{0.1}+3 y_{0.1} x_{2.0}{ }^{2}+3 y_{2.1} x_{1.0}{ }^{2}-y_{1.0} x_{3.0} x_{0.1} .
\end{aligned}
$$

Коэффициент при 1:

$$
a_{11}=-y_{1.0} x_{3.0} x_{1.0}+y_{3.0} x_{1.0}{ }^{2}+3 y_{1.0} x_{2.0}{ }^{2}-3 y_{2.0} x_{1.0} x_{2.0} \text {. }
$$

Формула (2.3) вместе с совокупностью формул (2.4)-(2.15) определяет закон преобразования третьей производной $y^{\prime \prime \prime}$ при точечных преобразованиях (2.1). 
3. Замкнутые классы уравнений (1.1). Будем искать инвариантный класс уравнений (1.1) с рациональной по $y^{\prime}$ и $y^{\prime \prime}$ правой частью. Воспользуемся правилом преобразования третьей производной (2.4)-(2.15) для простейшего уравнения вида (1.1)

$$
y^{\prime \prime \prime}=0 \text {. }
$$

Вид преобразованного уравнения (3.1)

$$
\begin{aligned}
\tilde{y}^{\prime \prime \prime} & =\frac{3 x_{0.1} \tilde{y}^{\prime \prime 2}}{x_{1.0}+x_{0.1} \tilde{y}^{\prime}} \\
& +\frac{a_{3} \tilde{y}^{\prime \prime} \tilde{y}^{\prime 2}+a_{4} \tilde{y}^{\prime \prime} \tilde{y}^{\prime}+a_{5} \tilde{y}^{\prime \prime}+a_{6} \tilde{y}^{\prime 5}+a_{7} \tilde{y}^{\prime 4}+a_{8} \tilde{y}^{\prime 3}+a_{9} \tilde{y}^{\prime 2}+a_{10} \tilde{y}^{\prime}+a_{11}}{\left(x_{1.0}+x_{0.1} \tilde{y}^{\prime}\right)\left(y_{1.0} x_{0.1}-x_{1.0} y_{0.1}\right)}
\end{aligned}
$$

позволяет искать замкнутый класс уравнений (1.1) в виде

$$
\begin{aligned}
y^{\prime \prime \prime}= & \frac{B(x, y) y^{\prime \prime 2}+P(x, y) y^{\prime \prime} y^{\prime 2}+Q(x, y) y^{\prime \prime} y^{\prime}+R(x, y) y^{\prime \prime}+S(x, y) y^{\prime 5}}{Y(x, y)-X(x, y) y^{\prime}} \\
& +\frac{L(x, y) y^{\prime 4}+K(x, y) y^{\prime 3}+M(x, y) y^{\prime 2}+N(x, y) y^{\prime}+T(x, y)}{Y(x, y)-X(x, y) y^{\prime}} .
\end{aligned}
$$

Преобразуем произвольное уравнение (3.3) с помощью формул (2.2)-(2.15). Модифицированное уравнение, вообще говоря, не принадлежит классу уравнений (3.3). Коэффициент $B /\left(Y-X y^{\prime}\right)$ при $y^{\prime \prime 2}$ в правой части (3.3) при преобразовании координат образует новый коэффициент при $y^{\prime} y^{\prime \prime 2}$ :

$$
\tilde{y}^{\prime \prime \prime}=\frac{\left(\widetilde{B}_{1}+\widetilde{B}_{2} \tilde{y}^{\prime}\right) \tilde{y}^{\prime \prime 2}}{\left(\widetilde{Y}-\widetilde{X} \tilde{y}^{\prime}\right)\left(x_{1.0}+x_{0.1} \tilde{y}\right)}+\cdots .
$$

Введем новую функцию $f(z)$, зависящую от параметра $z=y^{\prime}$, ассоциированную с уравнением (3.4):

где

$$
f(z)=\frac{\widetilde{B}_{1}+\widetilde{B}_{2} z}{x_{1.0}+x_{0.1} z}
$$

$$
\begin{aligned}
& \widetilde{B}_{1}=3 x_{0.1}\left(Y x_{0.1}-X y_{0.1}\right), \\
& \widetilde{B}_{2}=3 x_{0.1}\left(Y x_{1.0}-X y_{1.0}\right)+B\left(x_{1.0} y_{0.1}-x_{0.1} y_{1.0}\right) .
\end{aligned}
$$

Рациональная функция $f(z)$ из (3.5) имеет полюс первого порядка в точке $z_{0}=$ $-x_{1.0} / x_{0.1}$. Найдем вычет этой функции в точке $z_{0}$ и обозначим его через $\Omega$ :

$$
\Omega=\operatorname{Res}_{z=z_{0}} f(z) .
$$

Используя выражения (3.6), нетрудно получить явную формулу для величины $\Omega$, определенной в (3.7):

$$
\Omega=(B+3 X) \operatorname{det} S .
$$

Условие $\Omega=0$ составляет необходимое условие инвариантности класса уравнений (3.2). Точечные преобразования (1.2) невырождены, поэтому условие (3.8) эквивалентно дополнительному соотношению на функции $B$ и $X$

$$
B=-3 X \text {. }
$$

Непосредственной проверкой доказьвается, что условие (3.9) является также достаточным условием замкнутости класса уравнений (3.2) относительно точечных преобразований (1.2). Таким образом, доказана 
ТЕОРемА 1. Класс уравнений вида

$$
\begin{gathered}
y^{\prime \prime \prime}=\frac{-3 X(x, y) y^{\prime \prime 2}+P(x, y) y^{\prime \prime} y^{2}+Q(x, y) y^{\prime \prime} y^{\prime}+R(x, y) y^{\prime \prime}+S(x, y) y^{\prime 5}}{Y(x, y)-X(x, y) y^{\prime}} \\
+\frac{L(x, y) y^{\prime 4}+K(x, y) y^{\prime 3}+M(x, y) y^{\prime 2}+N(x, y) y^{\prime}+T(x, y)}{Y(x, y)-X(x, y) y^{\prime}}
\end{gathered}
$$

является инвариантным классом относительно преобразований (1.2).

Этот результат можно обобщить, если вместо уравнений (3.3) рассмотреть болееширокий класс уравнений

$$
y^{\prime \prime \prime}=-\frac{3 X f_{2}\left(1 / y^{\prime}\right) y^{\prime \prime 2}}{Y-X y^{\prime}}+f_{1}\left(\frac{1}{y^{\prime}}\right) y^{\prime 2} y^{\prime \prime}+f_{0}\left(\frac{1}{y^{\prime}}\right) y^{\prime 5} .
$$

Рациональные функции $f_{2}(z), f_{1}(z)$ и $f_{0}(z)$ в $(3.11)$ имеют полюсы в точке $z_{0}=$ $-x_{1.0} / x_{0.1}$.

Аналогично предыдушим рассуждениям необходимыми и достаточными условиями замкнутости класса уравнений (3.11) являются:

$$
\operatorname{Res}_{z=z_{0}} f_{2}(z)=1, \quad \operatorname{Res}_{z=z_{0}} f_{1}(z)=0, \quad \operatorname{Res}_{z=z_{0}} f_{0}(z)=0 .
$$

ТЕОремА 2. Класс уравнений (3.11) с рациональными функииями $f_{i}(i=0,1,2)$, удовлетворяющими соотношениям (3.12), замкнут относительно преобразований (1.2).

ДокАЗАТЕЛЬСТВО теоремы проводится прямьми вычислениями с использованием правил преобразования производных (2.2)-(2.15).

Автор выражает благодарность Р. А. Шарипову за постановку задачи и интерес, проявленньй к работе.

\section{СПИСОК ЦИТИРОВАННОЙ ЛИТЕРАТУРЫ}

[1] Liouville R. Sur les invariants de certaines équations différentielles et sur leurs applications // J. École Polytechnique. 1889. V. 59. P. 7-76.

[2] Lie S. Vorlesungen über continuierliche Gruppen. Leipzig: Teubner Verlag, 1893.

[3] Lie S. Theorie der Transformationsgruppen III. Leipzig: Teubner Verlag, 1930.

[4] Tresse A. Sur les invariants différentes des groupes continus de transformations // Acta Math. 1894. V. 18. P. 1-88.

[5] Tresse A. Determination des invariants ponctuels de l'équation différentielle ordinaire de 2ème ordre: $y^{\prime \prime}=w\left(x, y, y^{\prime}\right) / /$ Preisschriften der fürstlichen Jablonowski'schen Gesellschaft. V. XXXII. Leipzig: Hirzel, 1896.

[6] Cartan E. Sur les variétés à connection projective // Bull. Math. Soc. France. 1924. V. 52. P. 205-241.

[7] Cartan E. Sur les variétés à connexion affine et la théorie de la relativité generalisée // Ann. École Norm. Sup. 1923. V. 40. P. 325-412; 1924. V. 41. P. 1-25; 1925. V. 42. P. 17-88.

[8] Cartan E. Sur les espaces à connexion conforme // Ann. Soc. Math. Pologne. 1923. V. 2. P. 171-221.

[9] Картан Э. Пространства аффинной, проективной и конформной связности. Волгоград: Платон, 1997.

[10] Thomsen G. Über die topologischen Invarianten der Differentialgleichung $y^{\prime \prime}=f(x, y) y^{\prime 3}+$ $g(x, y) y^{\prime 2}+h(x, y) y^{\prime}+k(x, y) / /$ Abh. Math. Sem. Hamburg. Univ. 1930. V. 7. P. 301-328. 
[11] Grissom C., Thompson G., Wilkens G. Linearisation of second-order ordinary differential equations via Cartan's equivalence method // J. Differential Equations. 1989. V. 77. P. 1-15.

[12] Ibragimov N. Kh. Elementary Lie Group Analysis and Ordinary Differential Equations. Wiley Series in Math. Methods in Practice. V. 4. England: Wiley, 1999.

[13] Дрюма В.С. Геометрическая теория нелинейных динамических систем. Препринт ИМ с ВЦ АН МССР. Кишинев, 1986.

[14] Дрюма В.С. О теории подмногообразий проективных пространств, определяемых дифференциальньми уравнениями // Сб. статей. Кишинев: ИМ с ВЦ АН МССР, 1989. С. 75-87.

[15] Дрюма В. С. Геометрические свойства многомерных нелинейных дифференциальных уравнений и фазовое пространство динамических систем с финслеровой метрикой // ТМФ. 1994. T. 99. № 2. C. 241-249.

[16] Bordag L. A., Dryuma V.S. Investigation of Dynamical Systems Using Tools of the Theory of Invariants and Projective Geometry // NTZ-Preprint 24/95. Leipzig, 1995; // E-print solv-int/9705006, 1997.

[17] Bordag L. A. Symmetries of the Painleve equations and the connection with projective differential geometry // 7th EWM Meeting Proceedings. Trieste, Italy: Hindawi Publishing Corporation, 1997. P. 145-159.

[18] Babich M. V., Bordag L. A. Projective differential geometrical structure ot the Painlevé equations // J. Differential Equations. 1999. V. 157. № 2. P. 452-485.

[19] Романовский Ю.Р. Вычисление локальных симметрий обькновенных дифференциальных уравнений второго порядка методом эквивалентности Картана. Рукопись.

[20] Bacso S., Matsumoto M. On Finsler spaces of Douglas type. A generalisation of the notion of Berwald space // Publ. Math. Debrecen. 1997. V. 51. № 3-4. P. 385-406.

[21] Гусятникова В.Н., Юмагужин В.А. Точечные преобразования и линеаризуемость обыкновенных дифференциальных уравнений второго порядка // Матем. заметки. 1991. Т. 49. № 1. C. $146-148$.

[22] Dmitrieva V. V., Sharipov R. A. On the point transformations for the second order differential equations // E-print solv-int/9703003, 1997.

[23] Sharipov R. A. On the point transformations for the equation $y^{\prime \prime}=P+3 Q y^{\prime}+3 R y^{\prime 2}+S y^{\prime 3}$ // E-print solv-int/9706003, 1997.

[24] Sharipov R. A. Effective procedure of point classification for the equations $y^{\prime \prime}=P+3 Q y^{\prime}+$ $3 R y^{\prime 2}+S y^{\prime 3} / /$ E-print math.DG/9802027, 1998.

[25] Mikhailov O. N., Sharipov R. A. On the point expansion for a certain class of differential equations of second order // E-print solv-int/9712001, 1997. 